\title{
Guest Editors' Introduction to the Special Section on CVPR Papers
}

\author{
Simon Baker, Jiri Matas, and Ramin Zabih
}

$\mathrm{T}$ HIS special section contains extended versions of the following award-winning papers from the 2007 IEEE Conference on Computer Vision and Pattern Recognition (CVPR 2007):

- Bastian Leibe, Konrad Schindler, Nico Cornelis, and Luc Van Gool, "Coupled Object Detection and Tracking from Static Cameras and Moving Vehicles," winner of the Best Paper Prize, sponsored by Siemens and ObjectVideo.

- Anat Levin, Alex Rav-Acha, and Dani Lischinski, "Spectral Matting," winner of the Best Paper Prize Honorable Mention, sponsored by Honeywell and Siemens.

- Oncel Tuzel, Fatih Porikli, and Peter Meer, "Pedestrian Detection via Classification on Riemannian Manifolds," winner of the Best Paper Prize Honorable Mention, sponsored by Honeywell and Siemens.

- Yuan Li, Haizhou Ai, Takayoshi Yamashita, Shihong Lao, and Masato Kawade, "Tracking in Low Frame Rate Video: A Cascade Particle Filter with Discriminative Observers of Different Life Spans," winner of the Best Student Paper Prize, sponsored by IBM and the International Journal of Computer Vision.

Of the roughly 1,250 papers submitted to CVPR 2007, 60 were accepted as orals. During the Area Chair meeting, the Area Chairs and Program Chairs generated a short list of eight papers to be considered for awards. These papers were all read carefully by a committee of five senior members of the vision community in order to obtain an independent evaluation. The award committee members did not serve as Area Chairs for CVPR 2007 and included past recipients of best paper awards at leading vision conferences. Each member of the awards committee ranked the papers and provided justification of the ranking to the Program Chairs. The Program Chairs made the final decisions based on the input of the awards committee. Fortunately, the opinions of the

- S. Baker is with Microsoft Research, One Microsoft Way, Redmond, WA 98052. E-mail: sbaker@microsoft.com.

- J. Matas is with the Center for Machine Perception, Department of Cybernetics, Faculty of Electrical Engineering, Czech Technical University, Technicka 2, 16627 Praha 6, Czech Republic.

E-mail: matas@cmp.felk.cvut.cz.

- R. Zabih is with the Computer Science Department, 4130 Upson Hall, Cornell University, Ithaca, NY 14583. E-mail: rdz@cs.cornell.edu.

For information on obtaining reprints of this article, please send e-mail to: tpami@computer.org. committee were remarkably consistent and this decision was probably the easiest made by the Program Chairs in the entire process.

The authors of the award-winning papers and honorable mentions were invited to submit an extended version of their paper as a journal submission. The papers were reviewed by expert reviewers in the field, following the usual TPAMI procedure. All of the papers were accepted, in most cases after relatively minor revisions.

In addition to the awards appearing in this special section, CVPR 2007 also presented the Longuet-Higgins Prize for fundamental contributions in computer vision that have withstood the test of time. The prize, sponsored by GE and Microsoft, was awarded to two papers that were published in CVPR 1997: "Training Support Vector Machines: An Application to Face Detection," by E. Osuna, R. Freund, and F. Girosi, and "Normalized Cuts and Image Segmentation," by J. Shi and J. Malik.

\author{
Simon Baker \\ Jiri Matas \\ Ramin Zabih \\ CVPR Program Chairs and Guest Editors
}

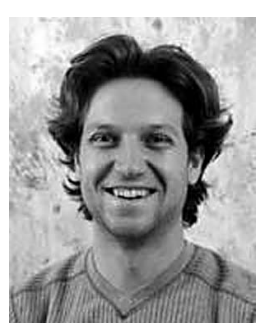

Simon Baker received the $\mathrm{PhD}$ degree from the Department of Computer Science at Columbia University in 1998, the MA degree in mathematics from Trinity College, Cambridge, in 1995, the MSc degree in computer science from the University of Edinburgh in 1992, and the BA degree in mathematics from Trinity College, Cambridge, in 1991. He is a senior researcher in the Interactive Visual Media Group at Microsoft Research Redmond (MSR). Before joining MSR, he was an associate research professor in the Robotics Institute at Carnegie Mellon University. His research interests include face recognition, modeling and tracking, human body modeling and tracking, super-resolution, 3D reconstruction, vision for safe driving, projectorcamera systems, and all aspects of video processing. Since 2004, he has been a associate editor for the IEEE Transactions on Pattern Analysis and Machine Intelligence. 


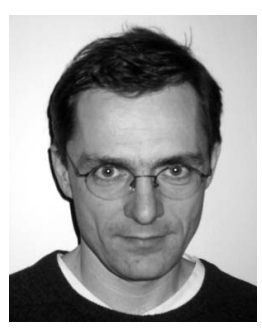

Jiri Matas received the MSc degree in cybernetics (with honors) from the Czech Technical University, Prague, Czechoslovakia, in 1987 and the PhD degree from the University of Surrey, United Kingdom, in 1995. From 1991 to 1997 , he was a research fellow at the Centre for Vision, Speech, and Signal Processing at the University of Surrey. In 1997, he joined the Center for Machine Perception at the Czech Technical University in Prague. Since 1997, he has held various positions at these two institutions. His research interests include object recognition, sequential pattern recognition, ensemble methods, invariant feature detection, and Hough Transform and RANSAC-type optimization. He received the best paper prize from the British Machine Vision Conferences in 2002 and 2005 and from the Asian Conference on Computer Vision in 2007. He has served as a program cochair for ECCV ' 04 and as a member of the editorial board of the International Journal of Computer Vision. Since 2006, he has been an associate editor for the IEEE Transactions on Pattern Analysis and Machine Intelligence.

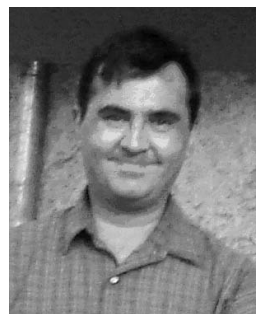

Ramin Zabih received the BS and MS degrees from the Massachusetts Institute of Technology and the PhD degree from Stanford University. $\mathrm{He}$ is a professor of computer science at Cornell University, Ithaca, New York, where he has twice received teaching awards. Since 2001, he has also held a joint appointment at Cornell's Weill Medical School. His research interests lie in early vision and its applications, with a focus on medicine. His work has made extensive use of discrete optimization techniques, especially graph cuts. Two papers he coauthored received the best paper prize from ECCV '02. In 2008, he co-organized the International Workshop on Computer Vision and gave a keynote speech at the British Machine Vision Conference. Since 2005, he has been an associate editor for the IEEE Transactions on Pattern Analysis and Machine Intelligence and, beginning in 2009, will serve as its editor-in-chief. 